\title{
Markenkommunikation auf Social Media- Plattformen - Anforderungen aus Konsumentensicht und deren Einfluss auf Zielgrössen des Markenmanagements
}
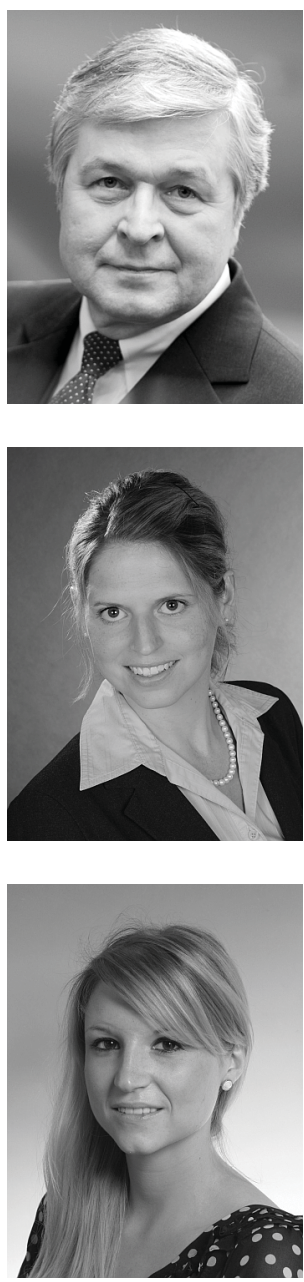

\section{Manfred Brubn, Daniela B. Schäfer und Verena Schoenmüller}

Markenkommunikation, Kommunikationsqualität, Social Media, Skalenentwicklung

Brand communication, communication quality, social media, scale development

Wenngleich die Kommunikation auf Social Media-Plattformen für viele Unternehmen bereits ein fester Bestandteil im Kommunikationsmix ist, sind den Markenmanagern die Anforderungen, die Konsumenten bei der Markenkommunikation auf Social Media-Plattformen an sie stellen, oftmals nicht bekannt. Die Ergebnisse unterstreichen die grosse Bedeutung der Erfüllung der inhaltlichen, beziehungsorientierten, interaktiven und multimedialen Anforderungen an die Qualität der Markenkommunikation auf Social Media-Plattformen. Zudem weisen die Resultate auf die Relevanz der Erfüllung dieser Anforderungen für die Erreichung von Zielgrössen des Markenmanagements hin, indem ein positiver Einfluss der Qualität der Markenkommunikation auf die Markeneinstellung und indirekt über die Markeneinstellung auf die Kaufabsicht belegt wird.

Communication on social media-platforms has become an inherent part of the companies' communication mix. However, very often marketers are not aware regarding the consumer demands concerning the brand communication on social media-platforms. The results highlight the great importance of fulfilling the content, relationship, interactivity, and multimedia related requirements in order to achieve a high quality of brand communication on social media platforms. Moreover, the findings point to the relevance of fulfilling these requirements for achieving marketing objectives by revealing that the brand's communication quality on social media-platform positively influences brand attitude and indirectly - through brand attitude - the purchase intention for the brand. 


\section{Einleitung}

„Social media is just a buzzword until you come up with a plan" (Zach Dunn - Mitgründer von One Mighty Roar).

Social Media-Plattformen erfahren seit einigen Jahren eine enorme Popularität im Internet. Weltweit partizipiert ein entscheidender Anteil der Bevölkerung an Social Media-Plattformen wie Facebook, Blogspot, YouTube, Wikipedia und Twitter. Die Social Media-Plattform Facebook zählt beispielsweise in der Schweiz 2,7 Millionen und in den USA 151,4 Millionen Mitglieder (Social Media Schweiz 2011). Auf dem Videoportal YouTube werden täglich 3 Milliarden Videos abgespielt (YouTube 2011). Somit bieten Social MediaPlattformen den Markenmanagern eine Plattform, die Aufmerksamkeit der Konsumenten auf die eigene Marke zu ziehen, in kurzer Zeit eine grosse Anzahl an Konsumenten zu erreichen, Kundenbeziehungen auf- und auszubauen sowie Weiterempfehlungen für die eigene Marke gezielt zu stimulieren. Diese endlos erscheinenden Möglichkeiten von Social Media-Plattformen führen zu einer sprunghaft ansteigenden Anzahl an Marken, die auf Social Media-Plattformen präsent sind. So hat die Marke Starbucks zum Beispiel mittlerweile über 20 Millionen Fans auf Facebook und über 1,3 Millionen Follower auf Twitter (Practical ecommerce 2011; Time 2011). Eine Studie der Universität Oldenburg und der Agentur construktiv (2009) bestätigt das steigende Bewusstsein von Unternehmen für Social Media-Kommunikation: 60 Prozent der 100 grössten Marken in Deutschland verfügen über einen Markenauftritt auf Social Media-Plattformen. Die Studie weist aber auch darauf hin, dass nur wenige Unternehmen eine Strategie ihrer Markenkommunikation auf Social Media-Plattformen definiert haben. Zudem ist nicht zu verkennen, dass nicht alle Marken mit ihrer Kommunikation auf Social Media-Plattformen erfolgreich sind. So ist die Marke Aldi bislang auf Social Media-Plattformen nahezu nicht vertreten und hat beispielsweise lediglich 5.000 Follower auf Twitter (Wolf 2011).

Somit stellt sich die Frage, warum manche Marken mit ihrer Kommunikation auf Social Media-Plattformen so erfolgreich sind, andere hingegen vergeblich versuchen, auf diesen Plattformen erfolgreich zu kommunizieren. Offensichtlich entsprechen nicht alle Kommunikationsstrategien den Anforderungen der Konsumenten. Dieses fehlende Wissen über die Konsumentenanforderungen ist jedoch für Marketingpraktiker höchst brisant, da die Kommunikation auf Social Media-Plattformen für die Wettbewerbsfähigkeit von Marken heute unabdingbar ist. Sie zählt neben Mediawerbung, Public Relations und Sponsoring längst zu einem festen Bestandteil des Kommunikationsmix. Die Folge einer qualitativ schlechten Markenkommunikation auf Social Media-Plattformen ist die Entstehung von Unzufriedenheit auf der Seite der Kommunikationsempfänger, verbunden mit einem Nichterreichen der Zielgrössen des Markenmanagements. Um dies zu vermeiden, ist die Kenntnis der Anforderungen, die Konsumenten im Rahmen der Kommunikation auf Social Media-Plattformen an Marken stellen, von Relevanz. Diese Kenntnis stellt die Basis für eine effektive Realisierung von Zielgrössen des Markenmanagements mithilfe der Social Media-Kommunikation dar. Für diejenigen Markenanbieter, die mit ihrer Markenkommunikation auf Social Media-Plattformen erfolgreich sind, bietet sie ein effizientes Kommunikationsinstrument.

Trotz der dargelegten Bedeutung der Konsumentenanforderungen an die Markenkommunikation auf Social Media-Plattformen existieren hierzu bislang keine Studien. Es liegen zwar Analysen zur Kommunikationsqualität in Face-to-Face- sowie virtuellen Interaktio- 
nen vor, diese befassen sich jedoch lediglich mit zwischenmenschlicher Kommunikation und lassen die Kommunikation zwischen Marke und Konsument sowie die spezifischen Charakteristika der Social Media-Plattformen als Kommunikationsumfeld ausser Acht. Das Ziel des vorliegenden Artikels ist daher, die Schliessung dieser Forschungslücke zu erreichen, indem diejenigen Anforderungen an die Markenkommunikation auf Social Media-Plattformen identifiziert werden, deren Erfüllung zu einer qualitativ hochwertigen Markenkommunikation auf Social Media-Plattformen führen. Zusätzlich wird die Bedeutung einer hohen Qualität der Markenkommunikation auf Social Media-Plattformen durch das Aufzeigen von deren Einfluss auf zentrale Zielgrössen des Markenmanagements ermittelt. Zur Erreichung der Ziele werden ein umfassender Literaturüberblick sowie eine quantitative Online-Umfrage mit 385 Schweizer Konsumenten durchgeführt.

Der Artikel gliedert sich in sechs Teile. In Kapitel 2 wird der Stand der Literatur zu den relevanten Forschungsfeldern aufgezeigt. Dies mündet in die Ableitung der zentralen Forschungsfragen. In Kapitel 3 wird eine Definition der Qualität der Markenkommunikation auf Social Media-Plattformen erarbeitet. Kapitel 4 widmet sich der Identifikation der Anforderungen an die Qualität der Markenkommunikation auf Social Media-Plattformen. Hierzu wird eine theorie- und sachlogischbasierte Konzeptualisierung vorgenommen. In Kapitel 5 erfolgt die Entwicklung eines Untersuchungsmodells zum Einfluss der Markenkommunikationsqualität auf Social Media-Plattformen auf Zielgrössen des Markenmanagements. Zudem erfolgt die quantitative Prüfung des Mess- sowie Untersuchungsmodells, um die Relevanz der Erfüllung der identifizierten Anforderungen für Marketingpraktiker zu unterstreichen. Kapitel 6 bildet das Fazit mit der Ableitung von Praxisimplikationen und einer kritischen Würdigung der Untersuchungsergebnisse.

\section{Stand der Literatur}

Die vorliegende Studie knüpft an zwei Forschungsfelder an. Zum einen an die Forschung zur Kommunikationsqualität und zum anderen an die Qualität von Social Media-Plattformen. Kommunikation kann Face-to-Face oder virtuell stattfinden. Somit lässt sich das Forschungsfeld zur Kommunikationsqualität weiter in die Face-to-Face- und die virtuelle Kommunikation aufgliedern. Die Face-to-Face-Kommunikation wird durch die körperliche Anwesenheit der Kommunikationspartner und durch den Einsatz von sämtlichen Sinnen und Kanälen charakterisiert (Daft/Lengel 1984; Rothe 2006; Geile 2010). Die auf dieses Forschungsfeld bezogenen Ergebnisse zeigen, dass sich die Qualität der Face-to-FaceKommunikation sowohl aus einer fachlichen als auch einer persönlichen Komponente zusammensetzt. Weiterhin demonstrieren sie, dass die fachliche Komponente durch Faktoren wie die Bedeutsamkeit und Nachvollziehbarkeit und die persönliche Komponente durch Faktoren wie die Vertrauenswürdigkeit, das Einfühlungsvermögen und die Gesprächsbereitschaft beschrieben werden (Shelby 1994; Mohr/Sohi 1995; Mohr/Spekman 1996; Shelby 1998; den Haring/Mattsson 1999; Sengupta/Krapfel/Pusateri 2000; Frommeyer 2005). Die Gesamtheit dieser Forschungsarbeiten bezieht sich auf die interpersonelle Kommunikation, bei der sich zwei Personen zur identischen Zeit am identischen Ort treffen. Da im vorliegenden Untersuchungsgegenstand die Kommunikation jedoch zwischen Marken und Konsumenten und zudem auf Social Media-Plattformen stattfindet, bieten die Resultate der Face-to-Face-Forschung für die vorliegende Studie lediglich erste Hinweise.

Unter virtueller Kommunikation wird die Kommunikation in einer computervermittelten Umgebung mittels so genannter „computer-mediated communication“ (CMC) verstan- 
den (Bordia 1997; Etzioni/Etzioni 1999). CMC wird auch als elektronische Kommunikation bezeichnet (Duckek 2010). Konkret wird der Begriff aufgefasst als Kommunikationsart, bei der Computer als Vermittlungsinstanzen zwischen Sender und Empfänger genutzt werden (Culnan/Markus 1987; Duckek 2010). Die Kommunikationsqualität in virtueller Umgebung ist Gegenstandsbereich der Forschungen von Lowry et al. (2006) und Duckek (2010). Lowry et al. (2006) untersuchen die Qualität der Kommunikation im Hinblick auf die Prozesszufriedenheit in virtuellen Arbeitsgruppen. Sie definieren die Kommunikationsqualität anhand der drei Dimensionen Kommunikationsoffenheit (Roberts/O'Reilly 1974; O’Reilly/Roberts 1977; Lowry et al. 2006), Diskussionseffizienz (Davison 1997; Lowry et al. 2006) und Effektivität der Aufgabenbesprechung (Gouran et al. 1978; Lowry et al. 2006). Duckek (2010) analysiert die Kommunikationsqualität in elektronischen Verhandlungen und definiert diese als das „Ausmaß erreichter gegenseitiger Verständigung“ (Duckek 2010, S. 127). Auch diese Definitionen und Ergebnisse zur Kommunikationsqualität lassen sich nicht uneingeschränkt auf den vorliegenden Untersuchungsgegenstand übertragen, da die Kommunikation im vorliegenden Fall zwar virtuell, jedoch unter den spezifischen Bedingungen von Social Media-Plattformen stattfindet und somit nicht interpersonell, sondern webbasiert zwischen Konsument und Marke verläuft. Darüber hinaus betrachten die dargestellten Forschungsarbeiten die Kommunikation vorwiegend im organisationalen Kontext, während die vorliegende Untersuchung die Kommunikation zwischen Konsumenten und der Marke bzw. der Kommunikation des Unternehmens in Bezug auf die Marke betrachtet.

Um den dargestellten Erkenntnisstand im Hinblick auf die spezifischen Bedingungen der Kommunikation auf Social Media-Plattformen zu ergänzen, bedarf es zudem eines Literaturüberblicks zur Qualität von Social Media-Plattformen, um den Besonderheiten des vorliegenden Untersuchungskontextes Rechnung zu tragen. Hinsichtlich der Qualität von Social Media-Plattformen liegen bereits einige Erkenntnisse vor. Hierbei fokussieren sich zahlreiche Forschungsarbeiten auf spezifische Kategorien von Social Media-Plattformen ${ }^{1}$ (Zeithaml et al. 2000; Schau/Gilly 2003; Ward/Ostrom 2006; Vickery/Wunsch-Vincent 2007; Chu/Kamal 2008; Trusov et al. 2009, 2010). So zeigen die empirischen Resultate, dass beispielsweise die Informationsrelevanz und -qualität, die Benutzerfreundlichkeit und Zuverlässigkeit zu einer qualitativ hochwertigen Social Media-Plattform führen (Lin/Lee 2006; Lin 2008; French 2009; Hsieh et al. 2010). Hagel und Armstrong (2006) argumentieren, dass Online-Communities vier soziale Grundbedürfnisse befriedigen müssen, um erfolgreich zu sein. Hierzu gehören die Pflege von Interessen, das Eingehen von zwischenmenschlichen Beziehungen, das Ausleben von Fantasien und das Abwickeln bzw. Tauschen von Geschäften. Darüber hinaus betont Preece (2001), dass eine gute Benutzerfreundlichkeit eine zentrale Bedeutung für die Qualität aus Sicht der Konsumenten einnimmt. Diese umfasst zum Beispiel Feedback-Funktionen, die den Dialog fördern, eine verständliche, aber auch ästhetisch ansprechende Informationsdarstellung und eine einfache Zugänglichkeit der Informationen. Johnson (2001) identifiziert eine adäquate technische Unterstützung und Online-Kommunikationstechniken als wichtige Anforderungen für den Erfolg von Online-Communities.

1 Die Social Media-Plattformen werden von den Autoren Kaplan und Haenlein (2010) in die Kategorien Collaborative Projects, Blogs, Content Communities, Social Networking Sites, Virtual Worlds und Virtual Game Worlds unterteilt. 
Wie aus dem Literaturüberblick hervorgeht, ist fraglich, ob die identifizierten Anforderungen im Rahmen der Forschung zur Kommunikation umfassend sind, da sie lediglich eine Ableitung aus Erkenntnissen von Studien darstellen, die sich entweder auf Face-to-FaceKommunikation begrenzen oder aber im virtuellen Umfeld lediglich zwischenmenschliche Kommunikation analysieren. Auch die weiterführende Betrachtung der Qualität von Social Media-Plattformen demonstriert, dass bisher keine Erkenntnisse über die Anforderungen an Marken im Rahmen der Kommunikation auf Social Media-Plattformen vorliegen. Die Kommunikation zwischen Marke und Konsument wird bislang somit vollständig ausser Acht gelassen. Spezifischer ausgedrückt liegt bislang keine Studie vor, die zum Ziel hat, die zentralen Konsumentenanforderungen an Marken im Rahmen der Kommunikation auf Social Media-Plattformen zu ermitteln.

Diese Forschungslücken geben an dieser Stelle Anlass, folgende Forschungsfragen abzuleiten, die nachfolgend beantwortet werden sollen:

(1) „Welches sind die Anforderungen, die Konsumenten im Rabmen der Kommunikation auf Social Media-Plattformen an Marken stellen?"

$\rightarrow$ Identifikation von Anforderungen (Kapitel 4)

(2) „Welche Relevanz kommt der Erfüllung dieser Anforderungen für die Erreichung von zentralen Zielgrössen des Markenmanagements zu?“

$\rightarrow$ Ermittlung der Relevanz der Anforderungserfüllung (Kapitel 5)

\section{Definition der Kommunikationsqualität von Marken auf Social Media-Plattformen}

Die Aufarbeitung der Literatur macht deutlich, dass bislang keine spezifische Begriffsauffassung für die Kommunikationsqualität von Marken auf Social Media-Plattformen existiert. Dies geht mit der Notwendigkeit einher, eine Definition zu erarbeiten. Hierfür erfolgt zunächst in Anlehnung an Frommeyer (2005) die Einteilung des Untersuchungsgegenstandes in verschiedene Gegenstandsbereiche: Untersuchungsbereich, instrumentelle Ebene, Bezugsobjekt und Perspektive. Hinsichtlich des Untersuchungsbereichs ist die Qualität der Markenkommunikation auf Social Media-Plattformen auf den Markt ausgerichtet und damit unternehmensextern. Die Qualität der Marketingkommunikation auf Social MediaPlattformen ist auf instrumenteller Ebene sowohl eine Dialogkommunikation, da sie durch die Eigenschaft der Interaktivität des Social Web zweiseitig ist, als auch eine Marketingkommunikation, da die Qualität letztlich über den Absatz eruiert wird. Aufgrund des spezifischen Kontextes findet die Kommunikation auf instrumenteller Ebene zudem online bzw. genauer ausgedrückt auf online Social Media-Plattformen statt. Diese Kommunikation auf Social Media-Plattformen weist Besonderheiten auf: Erstens ist die Reaktion der Konsumenten nicht uneingeschränkt kontrollierbar, aber öffentlich zugänglich. Damit einher geht, zweitens, dass es sich um ein interaktives Kommunikationsinstrument handelt. Drittens vollzieht sich die Kommunikation mittels Wort, Bild, Audio und Video. Viertens ist die Informationsdiffusion einfach, schnell, kostengünstig und kann, fünftens, sowohl persönlich als auch unpersönlich erfolgen (Brubn 2011). Im vorliegenden Fall definiert sich das Bezugsobjekt durch den Markenanbieter als Sender der Kommunikation. Schliesslich ist der Konsument derjenige, der die Qualität beurteilt und damit die Perspektive des Informationsempfängers für die Qualitätsbewertung einnimmt (siehe Abbildung 1). 


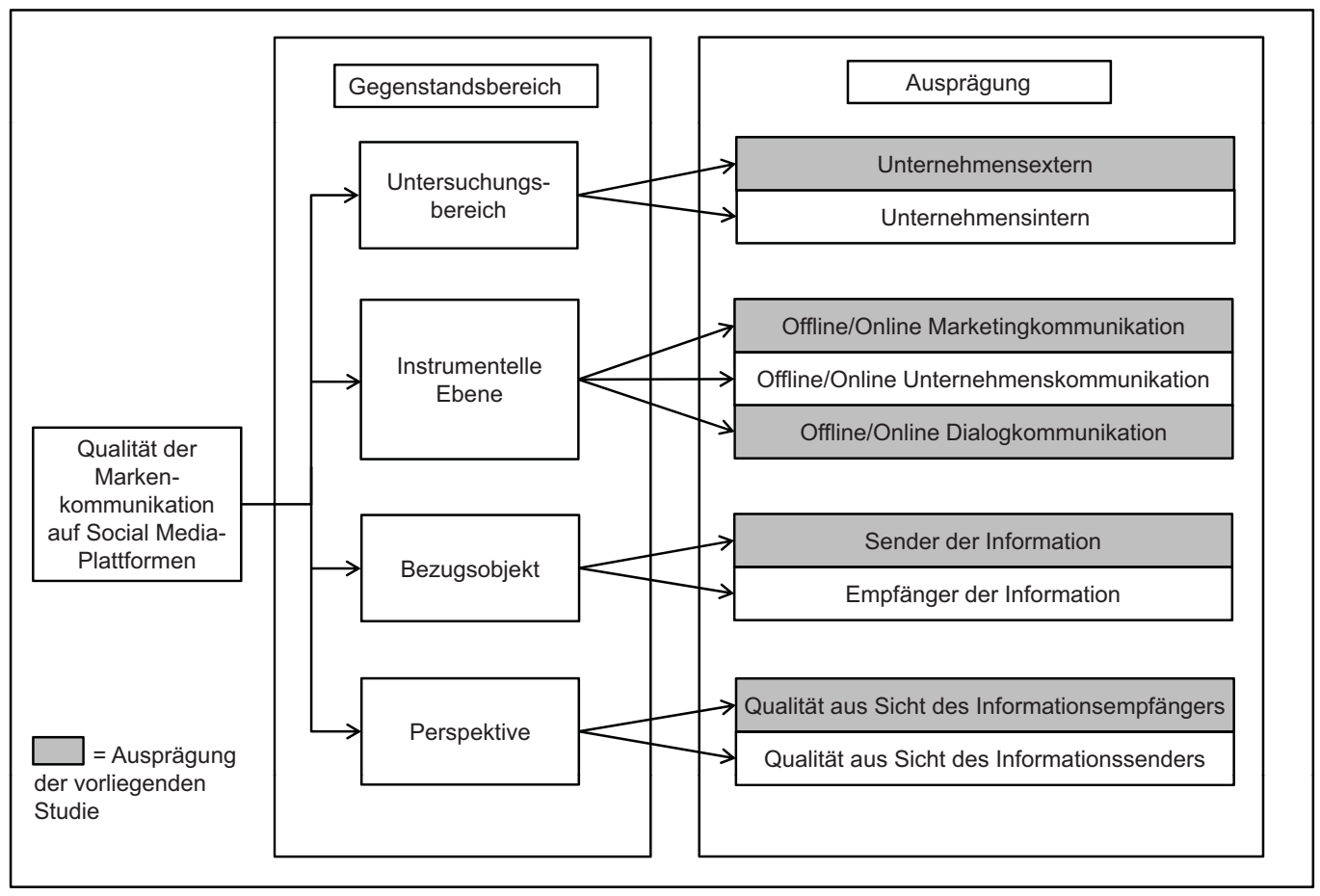

Abbildung 1: Gegenstandsbereiche der Qualität der Markenkommunikation auf Social Media-Plattformen

Dies führt zusammenfassend zu folgender Definition:

Die Qualität der Markenkommunikation auf Social Media-Plattformen ist die Fähigkeit des Markenanbieters, die Kommunikation auf Social Media-Plattformen gemäss den Konsumentenerwartungen auf einem bestimmen Anforderungsniveau zu erstellen.

\section{Identifikation von Anforderungen an die Kommunikationsqualität der Markenkommunikation auf Social Media-Plattformen}

Gemäss der Theorie des Interactional View (Watzlawick et al. 2003) beinhaltet die zwischenmenschliche Kommunikation sowohl einen Inhalts- als auch einen Beziehungsaspekt. Der Inhaltsaspekt gibt Auskunft darüber, „Was?“ übermittelt wird und stellt somit die Sachebene der Kommunikation dar (Plöttner 1999). Der Beziehungsaspekt bezieht sich darauf, „Wie?" kommuniziert wird und betont die Beziehungsebene zwischen den Kommunikationspartnern (Plöttner 1999). Eine hohe Kommunikationsqualität kann sich entsprechend dieser Theorie somit zum einen auf den Aspekt des Inhalts und zum anderen auf den Aspekt der Beziehung beziehen.

Die Theorie des Interactional View, die ursprünglich auf die zwischenmenschliche Kommunikation Bezug nimmt, lässt sich im Kontext des Konsumentenverhaltens auf die Kommunikation zwischen Personen und Objekten übertragen. Einen Erklärungsrahmen hierzu liefert die von Gilmore (1919) entwickelte Theorie des Animismus. Nach dieser Theorie neigen Menschen zu Anthropomorphismus, dem Verleihen von menschlichen Eigenschaf- 
ten auf nicht-lebende Objekte. Gemäss der Animismustheorie existieren im Hinblick auf die Vermenschlichung von Marken die drei Möglichkeiten „Repräsentation durch eine Person“, „persönliche Assoziationen“ und „Übertragung von Charaktereigenschaften“ (Fournier 1998). Aufgrund dieser theoretisch begründeten Vermenschlichung von Marken ist die Theorie des Interactional View auch auf den kommunikativen Austausch zwischen Konsument und Marke übertragbar.

Die spezifische Kommunikationsumgebung des Social Web macht jedoch zudem eine Erweiterung der Theorie des Interactional View notwendig. Entsprechend der Theorie des Interactional View stellt die Interaktivität einen weiteren Bestandteil der interpersonellen Kommunikation dar, betrachtet diese jedoch nicht als eigenständige Komponente. Um der Bedeutung der Interaktivität als konstitutives Merkmal der Social Media-Kommunikation im Vergleich zu anderen Kommunikationsinstrumenten (Kapitel 3) gerecht zu werden, bedarf es der Betrachtung dieser als eigenständige Komponente. Brexendorf (2010) betrachtet die Interaktivität im Kontext der Face-to-Face-Kommunikation aus drei möglichen Perspektiven und bezieht sich dabei auf die Theorien von Merten (1977) und Wabren (1987): Erstens, Interaktivität entspricht der interpersonellen Kommunikation. Zweitens, Interaktivität ist eine Determinante der interpersonellen Kommunikation. Drittens, interpersonelle Kommunikation ist ein Element der Interaktivität. Bezogen auf die Kommunikation auf Social Media-Plattformen bleibt hierbei unberücksichtigt, dass Social Media-Plattformen die Möglichkeit bieten, auch zeitverzögert, mit geringer Kontrolle sowie einseitig zu kommunizieren (Lowry et al. 2006). Zudem zeigen Ergebnisse der Forschung zur Qualität von Webseiten bzw. Online Retailing-Plattformen, dass die Interaktivität eine zentrale Komponente der Qualität für Konsumenten darstellt (Alpar 2001; Schlosser/Kanfer 2001; Srinivasan et al. 2002; Loiacono et al. 2007). Für die Kommunikation auf Social Media-Plattformen stellen diese Ergebnisse aufgrund der Besonderheiten der Kommunikationsplattform relevante Erkenntnisse für die Qualität der Kommunikation dar. Daher ist im Kontext der Markenkommunikation auf Social Media-Plattformen die Interaktivität als eine Komponente der Qualität der Kommunikation aufzufassen. So erweitert die vorliegende Studie die bisherigen Perspektiven aus Sicht der interpersonellen Kommunikation und sieht die Interaktivität als einen Bestandteil der Markenkommunikation auf Social Media-Plattformen an.

Ein weiterer spezifischer Aspekt der Markenkommunikation auf Social Media-Plattformen besteht in den Gestaltungsmöglichkeiten des Markenauftritts auf Social Media-Plattformen. Mittels verschiedener Medien, wie Videos, Graphiken oder Audiosequenzen, sind eine Vielzahl medialer Stimuli zur Kommunikation einsetzbar. Die Notwendigkeit der Berücksichtigung der Multimedialität wird durch Studien im Kontext der CMC deutlich. Bisherige Studien zur Qualität von Online-Erscheinungen, wie der Qualität im Kontext von Webseiten, Online-Händlern oder E-Commerce-Plattformen, zeigen die Notwendigkeit der Integration von Elementen des Designs und der Nutzung auf (Liu/Arnett 2000; Yoo/ Donthu 2001; Wolfingbarer/Gilly 2003). Wolfingbarer und Gilly (2003) bestätigen in ihrer Studie beispielsweise, dass das Design der Webseite eine von vier Dimensionen der Qualität von Online-Händlern darstellt. Diese Dimension umfasst sowohl ästhetische Aspekte, wie die Verwendung von Graphiken, aber auch Nutzungsaspekte, wie die Einfachheit der Navigation. Da Social Media-Plattformen aufgrund der Besonderheiten der Kommunikation im Internet die gleichen Aspekte der medialen Darstellung umfassen, erscheint es notwendig, die Qualität der Markenkommunikation auf Social Media-Plattformen um 
die multimediale Darstellung zu erweitern. Ein effektiver Multimediaeinsatz ist demnach notwendig, um die Qualitätsanforderungen des Konsumenten zufriedenzustellen (Purao/Han 2000). Dies unterstreicht zudem die Bedeutung des Multimediaeinsatzes für den vorliegenden Untersuchungsgegenstand, da die Erfüllung von multimedialen Anforderungen zur Erreichung einer hochwertigen Kommunikationsqualität von Marken auf Social Media-Plattformen wichtig ist. Somit ist davon auszugehen, dass bei der Markenkommunikation auf Social Media-Plattformen nicht nur der Inhalt und die Beziehung, sondern ebenfalls die Interaktivität und der Einsatz von Multimedia eine Anforderung an die Kommunikationsqualität stellt. Insgesamt ist festzuhalten, dass die Kommunikationsqualität der Markenkommunikation auf Social Media-Plattformen durch die inhaltliche (Inhaltsaspekt), beziehungsorientierte (Beziehungsaspekt), interaktive (Interaktivitätsaspekt) und multimediale (Multimediaaspekt) Anforderungserfüllung beeinflusst wird. Somit gilt:

Hypothese 1a/b/cld: Eine Verbesserung (a) des Kommunikationsinhalts, (b) der Kommunikationsbeziehung, (c) der Kommunikationsinteraktivität und (d) der Kommunikationsmultimedialität erhöht die Qualität der Markenkommunikation auf Social MediaPlattformen.

\section{Entwicklung eines Strukturgleichungsmodells zu den Wirkungen der Qualität der Markenkommunikation auf Social Media-Plattformen}

\subsection{Konzeptualisierung und Hypothesengenerierung}

Die Identifikation der Anforderungen an die Markenkommunikation auf Social MediaPlattformen dient als Basis für die Beantwortung der zweiten Forschungsfrage. Hier gilt es zu prüfen, welche Relevanz der Erfüllung der identifizierten Anforderungen für die Erreichung von Zielgrössen des Markenmanagements zukommt. Für die Ermittlung der jeweiligen Relevanz wird ein Strukturgleichungsmodell entwickelt, das auf Basis einer quantitativen Umfrage einer empirischen Überprüfung unterzogen wird.

Die Markeneinstellung ist „eine eindimensionale Größe, die einen gelernten und relativ dauerhaften Zustand beschreibt, gegenüber einer Marke mehr oder weniger stark positiv oder negativ zu reagieren“ (Baumgarth 2008, 79). Somit stellt die Markeneinstellung eine subjektive, affektive Bewertung der Marke durch den Konsumenten dar (Petty/Cacioppo 1981). Die Kommunikation von Marken auf Social Media-Plattformen ist dabei als Teilelement des Leistungsangebots eines Markenanbieters aufzufassen, das neben weiteren markenbezogenen Elementen in einen psychischen Vergleichsprozess einfliesst und zur Bildung der Einstellung des Konsumenten gegenüber der Marke beiträgt. Untersuchungen haben gezeigt, dass z. B. die interpersonelle Kommunikation (Brexendorf 2010) sowie die Marketingkommunikation (Stern/Zaichkowsky 1991; Esser 1995; Liebl 2003) einen Einfluss auf die Einstellungsbildung gegenüber der Marke haben. Darüber hinaus weisen Poh/ Adam (2002) einen positiven Zusammenhang zwischen der Markenkommunikation auf der unternehmenseigenen Webseite und der Markeneinstellung nach. Sie zeigen somit, dass dieser Einfluss auch auf den Kontext des World Wide Web übertragbar ist. Basierend auf den vorangegangenen Ausführungen ist folglich von einem positiven Zusammenhang zwischen der Qualität der Markenkommunikation auf Social Media-Plattformen und der Markeneinstellung auszugehen.

Entsprechend der Einstellungs-Verhaltens-Hypothese (Miniard/Cohen 1979; Miniard et al. 1983) ist zudem ein positiver Einfluss der Markeneinstellung auf die markenbezogene 
Kaufabsicht zu vermuten. Unter der markenbezogenen Kaufabsicht wird ein geistiger Zustand verstanden, der den Plan eines Konsumenten widerspiegelt, eine festgelegte Menge eines Markenprodukts in einem festgelegten Zeitraum zu kaufen (Howard 1994). Hieraus folgt:

Hypothese 2: Je besser die vom Konsumenten wabrgenommene Qualität der Markenkommunikation auf Social Media-Plattformen, desto positiver ist die Einstellung des Konsumenten zur Marke.

Hypothese 3: Je positiver die Einstellung des Konsumenten zur Marke, desto höher ist seine markenbezogene Kaufabsicht.

Das hier entwickelte Strukturgleichungsmodell setzt die Erfüllung der identifizierten Anforderungen an die Markenkommunikationsqualität auf Social Media-Plattformen in Beziehung zur Markeneinstellung sowie zur markenbezogenen Kaufabsicht. Die Erfüllung der Anforderungen sowie die Markeneinstellung und markenbezogene Kaufabsicht entziehen sich einer direkten Messbarkeit (Bagozzi/Fornell 1982; Bagozzi/Philipps 1982). Es besteht daher die Notwendigkeit einer Konstruktoperationalisierung, indem es für jedes Konstrukt beobachtbare Indikatoren zu finden gilt (Churchill 1979; Homburg/Giering 1996). Die Messbarmachung der Konstrukte Kommunikationsinhalt, -beziehung, -interaktivität und -multimedialität erfolgt anhand von jeweils drei Fragen. Die Formulierung der Items erfolgt in Anlehnung an bestehende Operationalisierungen (siehe Tabelle A1 im Anhang). Die Konstrukte Kommunikationsinhalt, -beziehung, -interaktivität und -multimedialität werden somit eindimensional operationalisiert. Das Gesamtkonstrukt Kommunikationsqualität von Marken auf Social Media-Plattformen ist hingegen ein Konstrukt zweiter Ordnung mit den vier Dimensionen Kommunikationsinhalt, -beziehung, -interaktivität und -multimedialität und damit mehrdimensional. Die Mehrdimensionalität gilt nicht für die Markeneinstellung und die Kaufabsicht, da diese entsprechend der einschlägigen wissenschaftlichen Meinung eindimensionale Konstrukte darstellen. Die Operationalisierung der Markeneinstellung und markenbezogenen Kaufabsicht erfolgt auf Basis von in der Literatur etablierten Konstrukten (siehe Tabelle A1 im Anhang).

Die Qualität der Markenkommunikation auf Social Media-Plattformen stellt entsprechend der in Kapitel 4 hergeleiteten Konzeptualisierung ein formatives Konstrukt zweiter Ordnung mit den vier Dimensionen Kommunikationsinhalt, -beziehung, -interaktivität und -multimedialität dar. Es wird daher postuliert, dass z. B. die Erfüllung der multimedialen Anforderungen die Qualität der Markenkommunikation auf Social Media-Plattformen erhöht. Zudem sind die Dimensionen unabhängig voneinander. Schliesslich führt eine Veränderung einer Dimension nicht zur Veränderung aller Dimensionen, d. h. die Dimensionen korrelieren nicht zwingend miteinander (Chin 1998 a; Diamantopoulos/Winklhofer 2001; Jarvis et al. 2003; Chin et al. 2008). Es ist weiterhin anzumerken, dass sämtliche Dimensionen der Qualität der Markenkommunikation auf Social Media-Plattformen sowie die Markeneinstellung und Kaufabsicht reflektiv operationalisiert werden. Der Grund hierfür ist die Überlegung, dass die einzelnen Indikatoren die Konstrukte widerspiegeln. Zudem liegt die Annahme einer Abhängigkeit bzw. Korreliertheit der einzelnen Indikatoren eines Konstrukts zugrunde (Jarvis et al. 2003). Somit besteht das Messmodell Qualität der Markenkommunikation auf Social Media-Plattformen aus einem formativen Konstrukt zweiter Ordnung mit den reflektiven Dimensionen Kommunikationsinhalt, -beziehung, -interaktivität und -multimedialität. Das Strukturgleichungsmodell besteht aus dem 
Konstrukt der Markenkommunikationsqualität auf Social Media-Plattformen sowie den reflektiven Konstrukten erster Ordnung Markeneinstellung und Kaufabsicht.

Zusammenfassend wurden somit bislang ein Messmodell sowie ein Strukturgleichungsmodell entwickelt, die es im Folgenden im Rahmen einer quantitativen Analyse zu bestätigen und überprüfen gilt. Das Messmodell stellt zum einen die Konsumentenanforderungen an die Qualität der Markenkommunikation auf Social Media-Plattformen dar. Zum anderen umfasst das Strukturgleichungsmodell Zielgrössen des Markenmanagements, um die Bedeutung der Kommunikationsqualität auf Social Media-Plattformen für Marketingpraktiker zu unterstreichen.

\subsection{Konzeption und Ergebnisse der quantitativen Untersuchung}

\subsubsection{Design und Durchführung der quantitativen Untersuchung}

Die Validierung des entwickelten Konstrukts der Markenkommunikationsqualität auf Social Media-Plattformen sowie der Einfluss der Markenkommunikationsqualität auf die dargestellten Konsequenzen wird auf Basis einer empirischen Untersuchung vorgenommen. Die hierfür erforderlichen Daten wurden im Rahmen einer standardisierten OnlineBefragung ermittelt. Die Rekrutierung der Teilnehmer erfolgte vor allem über stark frequentierte Social Media-Plattformen. Die Probanden erhielten die Möglichkeit, zu Beginn des Fragebogens zwischen verschiedenen Marken diejenige zu wählen, die sie bereits auf Social Media-Plattformen wahrgenommen haben. Hierdurch wurde gewährleistet, dass jeder Proband eine tatsächliche Vorstellung bezüglich der Kommunikation der betreffenden Marke auf Social Media-Plattformen hat. Die ausgewählten Marken stammen aus der Tourismus-, Elektronik- und Bekleidungsbranche. Die Auswahl dieser Branchen begründet sich in der starken Präsenz dieser Marken auf Social Media-Plattformen und ihrer grossen Anhängerzahl auf diesen Plattformen. Darüber hinaus wurden Marken innerhalb dieser Branchen auch mittels einer qualitativen Vorstudie als besonders stark vertretene Marken auf Social Media-Plattformen identifiziert. Im Anschluss an die Auswahl der Marke beantworteten die Probanden Fragen zur Qualität der Markenkommunikation auf Social Media-Plattformen in Bezug auf die gewählte Marke. Hierbei wurde die Bewertung der konstruktbildenden Indikatoren entlang einer siebenstufigen Likert-Skala (von 1 = stimme voll und ganz zu bis 7 = stimme überhaupt nicht $\mathrm{zu}$ ) vorgenommen. Während der sechswöchigen Datenerhebungsphase konnten 385 vollständige Datensätze generiert werden. Die Stichprobe ist gekennzeichnet durch einen Frauenanteil von 62,3\% und einen Männeranteil von 37,7\%. Ferner zeigt die Analyse der Stichprobe, dass das Durchschnittsalter der Teilnehmer 24,6 Jahre beträgt.

\subsubsection{Operationalisierung und Validierung der Modellkonstrukte}

Zunächst erfolgte die Überprüfung der Messmodelle anhand der Analyse der individuellen Itemreliabilitäten sowie der Überprüfung der Konvergenz- und Diskriminanzvalidität auf Konstruktebene (Fornell/Cha 1994; White et al. 2003). Die Itemreliabilität wird mittels der Itemladung auf das entsprechende Konstrukt untersucht (Mathwick/Wiertz/de Ruyter 2007). Alle Ladungen grösser als 0,50 (Hulland 1999) werden hierbei als akzeptabel erachtet. Sämtliche Ladungen überschreiten diesen Wert mit Faktorladungen grösser 0,55. Tabelle A1 (Anhang) gibt eine Übersicht über alle Items sowie ihre entsprechenden Ladungen und t-Werte. Die Konvergenzvalidität der reflektiven Faktoren erster Ordnung wird 
anhand der Kriterien der Faktorreliabilität und der durchschnittlich erfassten Varianz überprüft (Fornell/Larcker 1981; Chin 1998 b). Die Werte der Faktorreliabilitäten überschreiten den empfohlenen Mindestwert von 0,70 (Nunally/Bernstein 1994). Auch die Werte der durchschnittlich erfassten Varianz überschreiten für alle Konstrukte den geforderten Mindestwert von 0,50 (Fornell/Larcker 1981). Lediglich das Konstrukt Kommunikationsinhalt verfehlt diesen Schwellenwert knapp. Ferner werden die Konstrukte mithilfe des Fornell-Larcker-Kriteriums auf Diskriminanzvalidität untersucht. Es zeigt sich, dass die durchschnittlich erfasste Varianz aller Faktoren grösser ist als die quadrierte Korrelation zwischen den Faktoren. Somit liegt Diskriminanzvalidität vor (siehe Tabelle A2 im Anhang).

Da im Rahmen der vorliegenden Untersuchung die unabhängigen und abhängigen Variablen von jeweils einer Person beurteilt wurden, besteht die Möglichkeit des Vorliegens eines Common-Method-Bias (CMB). Um sicherzustellen, dass kein CMB besteht, wurde die Stichprobe zunächst dem Harman's One-Factor-Test unterzogen (Harman 1967; Podsakoff/Organ 1986; Podsakoff et al. 2003). Es zeigt sich im Rahmen der dazugehörigen exploratorischen Faktorenanalyse, dass mehrere Faktoren extrahiert werden und kein Faktor einen Grossteil der Kovarianz erklärt. Insofern liegt die Vermutung nahe, dass kein CMB besteht. Da dieser Test zum Teil kritisiert wird (Greve 2006), wurde in einem weiteren Schritt ein so genannter Common Method Factor ermittelt. Hierbei wird eine weitere latente Variable in das Modell eingeführt, die ein First-Order Konstrukt, gemessen über alle Indikatoren des Modells, darstellt (Podsakoff et al. 2003). Es ist keine signifikante Verschlechterung des Modellfits festzustellen. Zudem zeigt sich, dass die quadrierten Ladungen des eingeführten Faktors erheblich geringer sind als die der übrigen Konstrukte (Substantive Factor Loadings). Dies bestätigt, dass kein CMB vorliegt (Liang et al. 2007).

Die Überprüfung des formativen Konstrukts zweiter Ordnung der Qualität der Markenkommunikation auf Social Media-Plattformen (H1) erfolgt unter Einbezug der gesamten Datenbasis. Für die Analyse wird das Analyseprogramm AMOS 17.0 eingesetzt. Formative Modelle sind jedoch statistisch unteridentifiziert (Bollen/Lennox 1991), weshalb die Operationalisierung mittels zweier reflektiver Indikatoren erfolgt (Jarvis et al. 2003). Die Operationalisierung anhand zweier theoretisch fundierter reflektiver Indikatoren wird von Jarvis et al. (2003) als die bevorzugte Methode der Operationalisierung bei formativen Konstrukten bestätigt. Im Unterschied zu reflektiven Messmodellen kann die Untersuchung der internen Konsistenz bei formativ gemessenen Konstrukten aufgrund der nicht notwendigen Abhängigkeit zwischen den Indikatoren eines Konstrukts nicht erfolgen. Daher wird die Messung der externen Validität empfohlen (Diamantopoulos/Winklhofer 2001; Jarvis et al. 2003). Damit wird überprüft, ob die formativen Indikatoren das Konstrukt angemessen abbilden.

Die Ergebnisse demonstrieren, dass zwischen der Qualität der Markenkommunikation auf Social Media-Plattformen und den vier Dimensionen Kommunikationsinhalt, -beziehung, -interaktivität und -multimedialität ein signifikanter Zusammenhang besteht: Kommunikationsinhalt $(\beta=0,16 ; t=2,03)$, Kommunikationsbeziehung $(\beta=0,39 ; t=6,67)$, Kommunikationsinteraktivität $(\beta=0,14 ; \mathrm{t}=2,81)$ und Kommunikationsmultimedialität $(\beta=0,32 ; t=5,16)$. Die vier Dimensionen erklären zudem 70 Prozent der Varianz der Qualität der Markenkommunikation auf Social Media-Plattformen. Somit demonstrieren sämtliche Ergebnisse, dass die externe Validität des Messmodells der Qualität der Markenkommunikation auf Social Media-Plattformen mit den zugrundeliegenden Dimensionen 
Kommunikationsinhalt, -beziehung, -interaktivität und -multimedialität angenommen werden kann. Daher wird Hypothese 1 bestätigt.

\subsubsection{Schätzung des Kausalmodells und Modellbeurteilung}

Im Rahmen des Kausalmodells zu den Konsequenzen der Qualität der Markenkommunikation auf Social Media-Plattformen erfolgte eine Überprüfung der Hypothesen 2 und 3 über die postulierten Beziehungszusammenhänge zwischen den Konstrukten. Die Beurteilung des Gesamtmodellfits basiert auf den globalen Gütemassen mit den Stand Alone-Anpassungsmassen $\chi^{2} / \mathrm{df}$, RMSEA, SRMR und den inkrementellen Anpassungsmassen CFI und TLI. Die Resultate zeigen, dass alle globalen Gütekriterien ( $\chi^{2} / \mathrm{df}=2,491$; RMSEA = 0,062; SRMR = 0,071; CFI = 0,960; TLI = 0,953) die geforderten Anspruchsniveaus erreichen (Homburg/Klarmann 2006).

Die Überprüfung der Hypothesen über die Wirkungsbeziehungen demonstriert, dass im vorliegenden Modell die Qualität der Markenkommunikation auf Social Media-Plattformen einen signifikanten Einfluss auf die Markeneinstellung $(\beta=0,62 ; t=13,10)$ ausübt. Weiterhin kann der positive Zusammenhang zwischen Markeneinstellung und Kaufabsicht $(\beta=0,84 ; t=22,84)$ bestätigt werden. Demnach werden die Hypothesen 2 und 3 angenommen. Abbildung 2 zeigt die Wirkungszusammenhänge im Überblick.

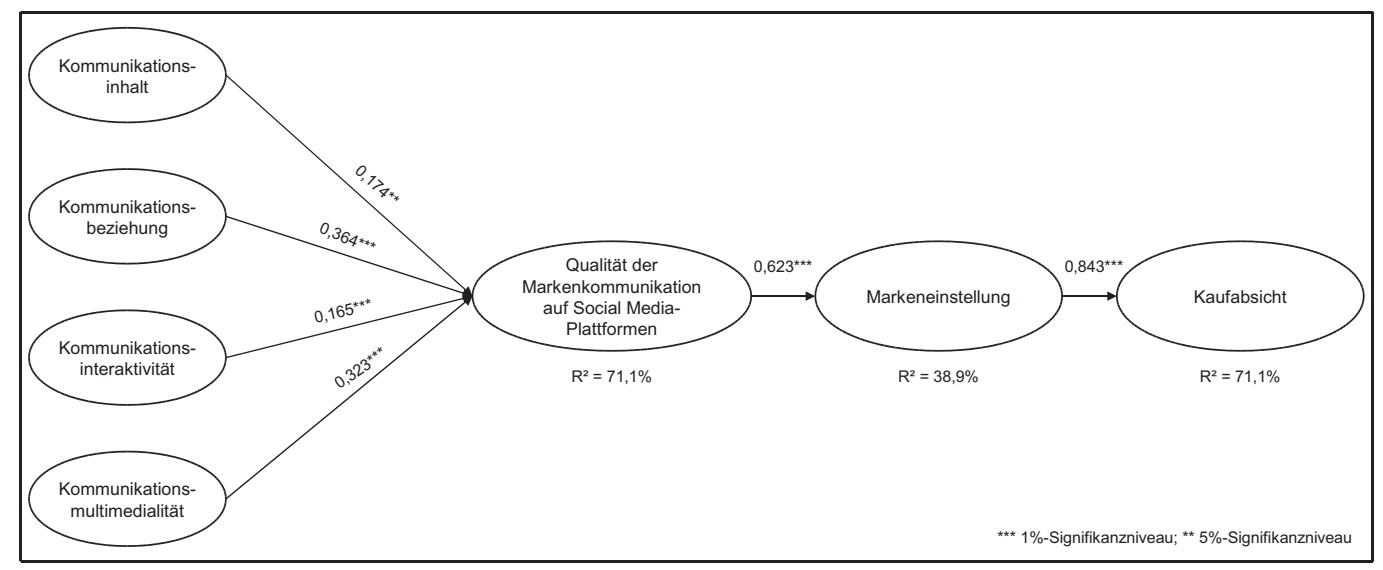

Abbildung 2: Ergebnisse des Strukturgleichungsmodells

\section{Fazit, Implikationen und kritische Würdigung}

Das Ziel dieses Artikels war es zum einen, diejenigen Anforderungen an die Markenkommunikation zu identifizieren, die Konsumenten im Rahmen der Kommunikation auf Social Media-Plattformen an Marken stellen. Zum anderen galt es als weitere Zielsetzung, die Relevanz der Erfüllung dieser Anforderungen für die Realisierung von zentralen Zielgrössen des Markenmanagements zu ermitteln. Für die Identifikation der Anforderungen wurde eine theoretische und sachlogische Konzeptualisierung durchgeführt. Dies führte zur Identifikation von Anforderungen an den Inhalt, die Beziehung, die Interaktivität und die Multimedialität der Kommunikation von Marken auf Social Media-Plattformen. Für die Ermittlung der Relevanz der Erfüllung dieser Anforderungen für die Erreichung von Ziel- 
grössen des Markenmanagements wurde eine quantitative Umfrage und darauf basierend eine Strukturgleichungsanalyse durchgeführt.

Die Ergebnisse demonstrieren, dass für eine erfolgreiche Markenkommunikation auf Social Media-Plattformen neben den klassischen Erfolgsfaktoren der Face-to-Face-Kommunikation - Kommunikationsinhalt und -beziehung -, auch für das Kommunikationsinstrument Social Media spezifische Anforderungen zu erfüllen sind. Die Resultate zeigen in diesem Zusammenhang, dass sowohl die Kommunikationsinteraktivität als auch die -multimedialität wesentlich zu einer qualitativ hochwertigen Markenkommunikation auf Social Media-Plattformen beitragen. Somit erklären die vier Dimensionen - Kommunikationsinhalt, -beziehung, -interaktivität und -multimedialität - das Zustandekommen einer hohen Markenkommunikationsqualität auf Social Media-Plattformen.

Eine genauere Betrachtung der Ergebnisse zeigt, dass der Kommunikationsbeziehung die grösste Bedeutung in Bezug auf die Qualität der Markenkommunikation auf Social MediaPlattformen zukommt. Auch die multimediale Darstellung übt einen vergleichsweise grossen Einfluss aus. Die Besonderheit des Kommunikationsinstruments Social Media zeigt sich in der im Vergleich zur Multimedialität geringeren Bedeutung des Kommunikationsinhalts für die Qualität der Markenkommunikation. Auch der Interaktivität kommt eine geringere Bedeutung zu. Dies ist möglicherweise darauf zurückzuführen, dass Konsumenten diese als Basisanforderungen an Social Media-Plattformen ansehen, die aufgrund des derzeitigen Technikstandes als gegeben betrachtet werden und damit deren Erfüllung die Qualität der Markenkommunikation nicht mehr erheblich steigern kann.

Für die Erfüllung der von Konsumenten gestellten beziehungsorientierten Anforderungen gilt es für Markenmanager, durch eine Auswahl geeigneter Mitarbeiter mit den entsprechenden Persönlichkeitsmerkmalen sowie regelmässige Mitarbeiterschulungen die Beziehungskompetenz der Mitarbeiter sicherzustellen. Zudem sind die Kommunikationssender aufgefordert, durch einen ehrlichen und glaubwürdigen Umgang mit den Kommunikationsempfängern, d. h. den Konsumenten, die Beziehung aufrechtzuerhalten und zu stärken. Für die Erfüllung der von Konsumenten gestellten multimedialen Anforderungen an die Markenkommunikation auf Social Media-Plattformen ist es, Markenmanagern anzuraten, den Einsatz von Text, Bild, Audio und Video so zu gestalten, dass die Markenkommunikation auf Social Media-Plattformen für den Empfänger ansprechend ist. Darüber hinaus bieten Social Media im Vergleich zu anderen Kommunikationsinstrumenten eine enorme Flexibilität in der Darstellung der Kommunikationsinhalte. Markenmanagern wird daher geraten, diese Vielfältigkeit in der Markenkommunikation auf Social MediaPlattformen zu nutzen und die Information in einem Text und/oder über gestaltetes Bildmaterial zu übermitteln sowie Audio- und Video-Streams zu integrieren. Für die Erfüllung der inhaltlichen Anforderungen empfiehlt es sich für Unternehmen, den Meinungs-, Informations- und Erfahrungsaustausch anzuregen und zu fördern. Dies erfordert eine entsprechende Inhaltskompetenz der Mitarbeiter in Bezug auf beispielsweise die Aktualität und Relevanz der Beiträge. Für die Erfüllung der interaktivitätsbezogenen Anforderungen gilt es zunächst, die Beteiligung der Konsumenten an der Kommunikation mit der Marke so einfach wie möglich zu gestalten. Dies kann auf Social Media-Plattformen vor allem mittels der optimalen Ausnutzung technischer Optionen, die auf diesen Plattformen angeboten werden, erzielt werden. So können als Beispiele die so genannte Pinnwand einer Markenprofilseite in dem sozialen Netzwerk Facebook oder aber der direkte Kontakt mit Mitarbeitenden des Unternehmens auf der Plattform Twitter angeführt werden. 
Die vorliegende Studie unterliegt auch einigen Limitationen. Das Modell zeigt zwar, inwieweit die Erfüllung der Anforderungen an die Markenkommunikation auf Social Media-Plattformen zur Verbesserung der Markeneinstellung und markenbezogenen Kaufabsicht beitragen, nicht aber, inwieweit sich diese auf das tatsächliche Kundenverhalten und ökonomische Grössen (z. B. Umsatz, Marktanteil) auswirkt. Eine Modellerweiterung ist somit künftig um diese Grössen vorzunehmen, so dass für Unternehmen der Beitrag der Social Media-Kommunikation quantitativ sichtbar gemacht werden kann. Im Rahmen der Studie wird sich ferner auf die Anforderungen von Konsumenten an die Markenkommunikation konzentriert. Dies stellt aber lediglich eine Fremdperspektive dar. Von Relevanz ist zudem die Ermittlung der Eigenperspektive. Hierfür ist das Markenmanagement nach Anforderungen, von denen es vermutet, dass diese von Konsumenten im Hinblick auf die Social Media-Kommunikation an sie gestellt werden, zu befragen. Zwischen der Eigen- und Fremdperspektive bestehen möglicherweise Abweichungen, die für Missverständnisse zwischen der Marke und den Konsumenten im Hinblick auf die Social Media-basierte Kommunikation ursächlich sein können. Weiterhin ist in zukünftigen Studien zu analysieren, ob Persönlichkeitseigenschaften der Konsumenten, wie z. B. die allgemeine Medienaffinität und Social Media-Erfahrung, die Wirkungszusammenhänge der Modelle beeinflussen. Der vorliegende Datensatz beinhaltet zudem überdurchschnittlich viele junge Probanden. Es ist anzunehmen, dass mit dem Einbezug von älteren Probanden, und damit einer breiteren Bevölkerungsschicht, die Überprüfung der postulierten Beziehungszusammenhänge zu anderen Resultaten kommt. Es ist z. B. zu vermuten, dass die Interaktivitätsqualität von älteren Konsumenten noch nicht als selbstverständlich angesehen wird und daher einen grösseren Einfluss auf die Qualität der Markenkommunikation auf Social Media-Plattformen hat. Dies ist in künftigen Studien zu überprüfen. Zudem beschränkt sich die Untersuchung auf Schweizer Konsumenten. Insofern ist die Studie auch auf internationaler Ebene durchzuführen, um daraus mögliche internationale Vergleiche zu ziehen.

\section{Literaturhinweise}

Alpar, P. (2001): Satisfaction with a Web Site: Its Measurement, Factors, and Correlates, Working Paper Nr. 99/01, Philipps-Universität Marburg, Institut für Wirtschaftsinformatik, Marburg.

Bagozzi, R.P./Fornell, C. (1982): Theoretical Concepts, Measurements, and Meaning, in: Fornell, C.

(Hrsg.): A Second Generation of Multivariate Analysis, 2. Aufl., New York, S. 24-38.

Bagozzi, R.P./Phillips, L.W. (1982): Representing and Testing Organizational Theories: A Holistic Construal, in: Administrative Science Quarterly, Jg. 27, Nr. 3, S. 459-489.

Baumgarth, C. (2008): Markenpolitik: Markenwirkungen - Markenführung - Markenforschung, 3. Aufl., Wiesbaden.

Bollen, K.A./Lennox, R. (1991): Conventional Wisdom on Measurement: A Structural Equation Perspective, in: Psychological Bulletin, Jg. 110, Nr. 2, S. 305-314.

Bordia, P. (1997): Face-to-Face Versus Computer-Mediated Communication: A Synthesis of the Experimental Literature, in: The Journal of Business Communication, Jg. 34, Nr. 1, S. 99-120.

Brexendorf, T.O. (2010): Markenloyalität durch persönliche Kommunikation. Eine dyadische Analyse von Verkäufer-Kunden-Interaktion am Beispiel der Marke BMW, Göttingen.

Brubn, M. (2011): Unternehmens- und Marketingkommunikation. Handbuch für ein integriertes Kommunikationsmanagement, 2. Aufl., München. 
Chattopadhyay, A./Basu, K. (1990): Humor in Advertising: The Moderating Role of Prior Brand Evaluation, in: Journal of Marketing Research, Jg., 27, Nr. 4, S. 466-476.

Chin, W.W. (1998 a): Issues and Opinion on Structural Equation Modeling, in: MIS Quarterly, Jg. 22, Nr. 1, S. 7-16.

Chin, W.W. (1998 b): The Partial Least Approach to Structural Equation Modeling, in: Marcoulides, G.A. (Hrsg.): Modern Methods for Business Research, Mahwah, S. 295-336.

Chin, W.W./Peterson, R.A./Brown, S.P. (2008): Structural Equation Modeling in Marketing - Some Practical Reminders, in: Journal of Marketing Theory and Practice, Jg. 16, Nr. 4, S. 278-298.

Chu, S.-C./Kamal, S. (2008): The Effect of Perceived Blogger Credibility and Argument Quality on Message Elaboration and Brand Attitudes: An Exploratory Study, in: Journal of Interactive Advertising, Jg. 8, Nr. 2, S. 26-37.

Churchill, G.A. (1979): A Paradigm for Developing Better Measures of Marketing Constructs, in: Journal of Marketing Research, Jg. 16, Nr. 1, S. 64-73.

Culnan, M.J./Markus, M.L. (1987): Information Technologies, in: Jablin, F.M./Putnam, L.L./ Roberts, K.H./Porter, L.W. (Hrsg.): Handbook of Organizational Communication. An Interdisciplinary Perspective, Newbury Park, S. 420-443.

Daft, R.L./Lengel, R.H. (1984): Information Richness: A New Approach to Managerial Behavior and Organizational Design, in: Research in Organizational Behavior, Jg. 6, Nr. 1, S. 191-233.

Davison, R.M. (1997): An Instrument for Measuring Meeting Success, in: Information and Management, Jg. 32, Nr. 4, S. 163-176.

den Haring, M./Mattsson, J. (1999): A Linguistic Approach to Studying Quality of Face-to Face Communication, in: The Service Industries Journal, Jg. 19, Nr. 2, S. 28-48.

Diamantopoulos, A./Winklhofer, H.M. (2001): Index Construction with Formative Indicators: An Alternative to Scale Development, in: Journal of Marketing Research, Jg. 38, Nr. 2, S. 269-277.

Dodds, W.B./Monroe, K.B./Grewal, D. (1991): Effects of Price, Brand, and Store Information on Buyers' Product Evaluations, in: Journal of Marketing Research, Jg. 28, Nr. 3, S. 307-319.

Duckek, K. (2010): Ökonomische Relevanz von Kommunikationsqualität in elektronischen Verhandlungen, Wiesbaden.

Esser, M. (1995): Werbeerfolgskontrolle auf Basis eines Modells der Werbewirkung, Frankfurt am Main u.a.

Etzioni, A./Etzioni, O. (1999): Face-to-Face and Computer-Mediated Communities: A Comparative Analysis, in: The Information Society, Jg. 15, Nr. 4, S. 241-248.

Fornell, C./Cha, J. (1994): Partial Least Squares, in: Bagozzi, R.P. (Hrsg.): Advanced Methods of Marketing Research, Cambridge, S. 52-78.

Fornell, C./Larcker, D.A. (1981): Evaluating Structural Equation Models with Unobservable Variables and Measurement Error, in: Journal of Marketing Research, Jg. 18, Nr. 1, S. 39-50.

Fournier, S.M. (1998): Consumers and Their Brands: Developing Relationship Theory in Consumer Research, in: Journal of Consumer Research, Jg. 24, Nr. 4, S. 343-373.

French, A.M. (2009): Social Networking Systems Success Model: Assessment and Validation of the IS Success Model in Social Context, Mississippi State University, Mississippi.

Frommeyer, A. (2005): Kommunikationsqualität in persönlichen Kundenbeziehungen. Konzeptualisierung und empirische Prüfung, Wiesbaden.

Geile, A. (2010): Face-to-Face Kommunikation im Vertrieb von Industriegütern, Wiesbaden.

Gilmore, G.W. (1919): Animism, Boston. 
Gouran, D.S./Brown, C.R./Henry, D.R. (1978): Behavioral Correlates of Perceptions of Quality in Decision-Making and Discussions, in: Communication Monographs, Jg. 45, Nr. 1, S. 51-63.

Greve, G. (2006): Erfolgsfaktoren von Customer-Relationship-Management-Implementierungen, Wiesbaden.

Hagel, J./Armstrong, G.A. (2006): Net Gain Profit im Netz, Heidelberg.

Harman, H.H. (1967): Modern Factor Analysis, Chicago.

Homburg, Ch./Giering, A. (1996): Konzeptualisierung und Operationalisierung komplexer Konstrukte - Ein Leitfaden für die Marketingforschung, in: Marketing ZFP, Jg. 18, Nr. 1, S. 5-24.

Howard, J. (1994): Buyer Behavior in Marketing Strategy, Englewood Cliffs.

Hsieh, C.-C./Kuo, P.-L./Yang, S.-C./Lin, S.-H. (2010): Assessing Blog-User Satisfaction Using the Expectation and Disconfirmation Approach, in: Computers in Human Behavior, Jg. 26, Nr. 6, S. 1434-1444.

Hulland, J. (1999): Use of Partial Least Squares (PLS) in Strategic Management Research: A Review of Four Recent Studies, in: Strategic Management Journal, Jg. 20, Nr. 2, S. 195-204

Jarvis, C.B./Mackenzie, S.B./Podsakoff, Ph.M./Mick, D.G./Bearden, W.O. (2003): A Critical Review of Construct Indicators and Measurement Model Misspecifications in Marketing and Consumer Research, in: Journal of Consumer Research, Jg. 30, Nr. 2, S. 199-218.

Johnson, C.M. (2001): A survey of current research on online communities of practice, in: The Internet and Higher Education, Jg. 30, Nr. 1, S. 45-60.

Kaplan, A.M./Haenlein, M. (2010): Users of the World, Unite! The Challenges and Opportunities of Social Media, in: Business Horizons, Jg. 53, Nr. 1, S. 59-68.

Liang, H./Saraf, N./Hu, Q./Xue, Y. (2007): Assimilation of Enterprise Systems: The Effect of Institutional Pressures and the Mediating Role of Top Management, in: MIS Quarterly, Jg. 31, Nr. 1, S. 59-87.

Liebl, C. (2003): Kommunikations-Controlling. Ein Beitrag zur Steuerung der Marketing-Kommunikation am Beispiel der Marke Mercedes-Benz, Wiesbaden.

Lin, H.-F. (2008): Determinants of Successful Virtual Communities: Contributions from System Characteristics and Social Factors, in: Information and Management, Jg. 45, Nr. 8, S. 522-527.

Lin, H.-F./Lee, G.G. (2006): Determinants of Success for Online Communities: An Empirical Study, in: Behavior and Information Technology, Jg. 25, Nr. 6, S. 479-488.

Liu, C./Arnett, K.P. (2000): Exploring the Factors Associated with Web Site Success in the Context of Electronic Commerce, in: Information \& Management, Jg. 38, Nr. 1, S. 23-33.

Loiacono, E.T./Watson, R.T./Goodhue, D.L. (2007): WEBQUAL: An Instrument for Consumer Evaluation of Websites, in: International Journal of Electronic Commerce, Jg. 11, Nr. 3, S. 51-87.

Lowry, P.B./Romano, N.C./Jenkins, J.L./Guthrie, R.W. (2006): The CMC Interactivity Model: How Interactivity Enhances Communication Quality and Process Satisfaction Lean-Media Groups, in: Journal of Management Information Systems, Jg. 26, Nr. 1, S. 155-195.

Mathwick, C./Wiertz, C./De Ruyter, K. (2008): Social Capital Production in a Virtual P3 Community, in: Journal of Consumer Research, Jg. 34, Nr. 6, S. 832-849.

McMillan, S.J./Hwang, J.S. (2002): Measures of Perceived Interactivity. An Exploration of the Role of Direction of Communication, User Control, and Time in Shaping Perceptions of Interactivity, in: Journal of Advertising, Jg. 31, Nr. 3, S. 29-42.

Merten, K. (1977): Kommunikation. Eine Begriffs- und Prozessanalyse, Opladen. 
Miniard, P./Cohen, J.P. (1979): Isolating Attitudinal and Normative Influences in Behavioral Intention Models, in: Journal of Marketing Research, Jg. 16, Nr. 1, S. 102-110.

Miniard, P.W./Obermiller, C./Page, T.J. (1983): A Further Assessment of Measurement Influences on the Intention-Behavior Relationship, in: Journal of Marketing Research, Jg. 20, Nr. 2, S. 206-213.

Mohr, J.J./Sohi, R.S. (1995): Communication Flows in Distribution Channels: Impact on Assessment of Communication Quality and Satisfaction, in: Journal of Retailing, Jg. 71, Nr. 4, S. 393-416.

Mohr, J.J./Spekman, R.E. (1996): Perfecting Partnerships: Several Characteristics Contribute to Successful Alliance between Chanel Members, in: Marketing Management, Jg. 4, Nr. 4, S. 35-43.

Nunnally, J.C./Bernstein I.H. (1994): Psychometric Theory, 3. Aufl., New York.

O’Reilly, C.A./Roberts, K.H. (1977): Task Group Structure, Communication, and Effectiveness in Three Organizations, in: Journal of Applied Psychology, Jg. 62, Nr. 6, S. 674-681.

Petty, R.E./Cacioppo, J.T. (1981): Attitudes and Persuasion: Classic and Contemporary Approaches, Dubuque.

Plöttner, O. (1999): Grundlagen der Gestaltung der Kommunikationsleistung, in: Kleinaltenkamp, M./Plinke, W. (Hrsg.): Geschäftsbeziehungsmanagement, Berlin, S. 1-62.

Podsakoff, N.P./Organ, D.W. (1986): Self-Reports in Organizational Research: Problems and Prospects, in: Journal of Management, Jg. 12, Nr. 5, S. 531-544.

Podsakoff, P.M./MacKenzie, S.B./Lee, J.-Y./Podsakoff, N.P. (2003): Common Method Biases in Behavioral Research: A Critical Review of the Literature and Recommended Remedies, in: Journal of Applied Psychology, Jg. 88, Nr. 5, S. 879-903.

Poh, D.M.H./Adam, S. (2002): An Exploratory Investigation of Attitude Toward the Website and the Advertising Hierarchy of Effects, in: Treloar, A./Ellis, A. (Hrsg.): AusWeb02, the Web Enabled Global Village: Proceedings of AusWeb02, the Eighth Australian World Wide Web Conference, Australien, S. 620-631.

Practical ecommerce (2011): 48 Facebook Pages for Businesses, http://www.practicalecommerce.com/articles/2667-48-Facebook-Pages-for-Businesses-, Zugriff: 10.10.2011.

Preece, J. (2001): Sociability and Usability in Online Communities: Determining and Measuring Success, in: Behaviour \& Information Technology, Jg. 20, Nr. 5, S. 347-356.

Purao, S./Han, T.-D. (2000): Distributing Multimedia Content to Balance Quality Service and Cost, in: Journal of Management Information Systems, Jg. 17, Nr. 1, S. 141-165.

Roberts, K.H./O'Reilly, C.A. (1974): Failures in Upward Communication in Organizations: Three Possible Culprits, in: Academy of Management Journal, Jg. 17, Nr. 2, S. 205-215.

Rothe, F. (2006): Zwischenmenschliche Kommunikation. Eine interdisziplinäre Grundlegung, Wiesbaden.

Schau, H.J./Gilly, M.C. (2003): We Are What We Post? Self-Presentation in Personal Web Space, in: Journal of Consumer Research, Jg. 30, Nr. 3, S. 385-404.

Schlosser, A.E./Kanfer, A. (2001): Interactivity in Commercial Web Sites: Implications for Web Site Effectiveness, $8^{\text {th }}$ Annual Conference of the Society for Consumer Psychology, February, Scottsdale, Arizona.

Sengupta, S./Krapfel, R.E./Pusateri, M.A. (2000): An Empirical Investigation of Key Account Sales Person Effectiveness, in: Journal of Personal Selling and Sales Management, Jg. 20, Nr. 4, S. 253-261. 
Shelby, A.N. (1994): Communication Quality as a Metacommunication: A Conceptual Analysis, in: van Waes, L./Woudstra, E./van den Hoven, P. (Hrsg.): Functional Communication Quality: Utrecht Studies in Language and Communication, Amsterdam, S. 5-16.

Shelby, A.N. (1998): Communication Quality Revisited: Exploring the Link with Persuasive Effects, in: The Journal of Business Communication, Jg. 35, Nr. 3, S. 387-404.

Social Media Schweiz (2011): Facebook: Die Welt im Überblick (Juni 2011), http://www.socialmediaschweiz.ch/Facebook_-_Die_Welt_Update_Juni_2011_.pdf, Zugriff: 14.10.2011.

Srinivasan, S.S./Anderson, R./Ponnavolu, K. (2002). Customer Loyalty in E-Commerce: An Exploration of its Antecedents and Consequences, in: Journal of Retailing, Jg. 78, Nr. 1, S. 41-50.

Stern, B./Zaichkowsky, J.L. (1991): The Impact of 'Entertaining' on Consumer Responses, in: Australian Marketing Researcher, Jg. 14, Nr. 1, S. 68-80.

Time (2011): 140 Best Twitter Feeds. Starbucks, http://www.time.com/time/specials/packages/ article/0,28804,2058946_2059086_2059073,00.html, Zugriff: 10.10.2011.

Trusov, M./Bodapati, A.V./Bucklin, R.E. (2010): Determining Influential Users in Internet Social Networks, in: Journal of Marketing Research, Jg. 47, Nr. 4, S. 643-658.

Trusov, M./Bucklin, E./Pauwels, K. (2009): Effects of Word-of-Mouth Versus Traditional Marketing: Findings from an Internet Social Networking Site, in: Journal of Marketing, Jg. 73, Nr. 5, S. 90-102.

Universität Oldenburg/construktiv (2009): Wie nutzen Deutschlands größte Marken Social Media? Eine empirische Studie, http://www.construktiv.de/newsroom/wp-content/uploads/2010/06/ construktiv-Social-Media-Studie.pdf, Zugriff: 14.10.2011.

Vickery, G./Wunsch-Vincent, S. (2007): Participative Web and User-Created Content. Web 2.0, Wikis and Social Networking, Paris.

Wabren, H.-K. (1987): Zwischenmenschliche Kommunikation und Interaktion in Unternehmen: Grundlagen, Probleme und Ansätze, Berlin.

Ward, J.C./Ostrom, A.L. (2006): Complaining to the Masses: The Role of Protest Framing in Customer-Created Complaint Web Sites, in: Journal of Consumer Research, Jg. 33, Nr. 2, S. 220-230.

Watzlawick, P./Beavin, J.H./Jackson, D.D. (2003): Menschliche Kommunikation. Formen - Störungen - Paradoxien, 10. Aufl., Bern.

White, C.J./Varadarajan, R./Dacin, P.A. (2003): Market Situation Interpretation and Response: The Role of Cognitive Style, Organizational Culture, and Information Use, in: Journal of Marketing, Jg. 67, Nr. 3, S. 63-79.

Wolf, M. (2011): Social Media Strategien im Vergleich: VW, eon, Mercedes, Deutsche Telekom, Aldi \& Lidl, http://michaelsevernotes.posterous.com/social-media-strategien-im-vergleich-vw-eonm, Zugriff: 14.10.2011.

Wolfinbarger, M./Gilly, M.C. (2003): eTailQ: Dimensionalizing, Measuring, and Predicting etail Quality, in: Journal of Retailing, Jg. 79, Nr. 3, S. 183-198.

Yoo, B./Donthu, N. (2001): Developing a Scale to Measure the Perceived Quality of an Internet Shopping Site (SITEQUAL), in: Quarterly Journal of Electronic Commerce, Jg. 2, Nr. 1, S. 31-46.

YouTube (2011): YouTube Fact Sheet, http://youtube-global.blogspot.com/2011/05/thanks-youtubecommunity-for-two-big.html, Zugriff: 14.10.2011.

Zack, M. (1993): Interactivity and Communication Mode Choice in Ongoing Management Groups, in: Information Systems Research, Jg. 4, Nr. 3, S. 207-239. 
Zeithaml, V.A./Parasuraman, A./Malhotra, A. (2000): A Conceptual Framework for Understanding E-Service Quality: Implications for Future Research and Managerial Practice, in: Marketing Science Institute Report, Nr. 00-115.

\section{Anhang}

\begin{tabular}{|c|c|c|}
\hline \multicolumn{3}{|c|}{ Kommunikationsinhalt (in Anlehnung an Frommeyer 2005; Geile 2010) } \\
\hline \multicolumn{3}{|l|}{ Faktorreliabilität $=0,72 ; \mathrm{C} \alpha=0,76 ; \mathrm{DEV}=0,47$} \\
\hline Items & Faktorladung & $\mathrm{t}$-Wert $(>1,64)$ \\
\hline $\begin{array}{l}\text { Die Inhalte der Beiträge von „Marke“ auf den Social } \\
\text { Media-Plattformen sind vollständig. }\end{array}$ & 0,625 & 11,852 \\
\hline $\begin{array}{l}\text { Die Inhalte der Beiträge von „Marke“ auf den Social } \\
\text { Media-Plattformen sind von Relevanz. }\end{array}$ & 0,725 & 13,595 \\
\hline $\begin{array}{l}\text { Ich finde, dass die Beitragsinhalte von „Marke“ auf den } \\
\text { Social Media-Plattformen verständlich sind. }\end{array}$ & 0,809 & 15,016 \\
\hline \multicolumn{3}{|c|}{ Kommunikationsbeziehung (in Anlehnung an Frommeyer 2005; Geile 2010) } \\
\hline \multicolumn{3}{|l|}{ Faktorreliabilität $=0,88 ; \mathrm{C} \alpha=0,88 ; \mathrm{DEV}=0,71$} \\
\hline Items & Faktorladung & $\mathrm{t}$-Wert $(>1,64)$ \\
\hline $\begin{array}{l}\text { Ich habe das Gefühl, dass sich „Marke“ auf den Social } \\
\text { Media-Plattformen bei der Kommunikation auch an } \\
\text { meinen Bedürfnissen orientiert. }\end{array}$ & 0,756 & 16,683 \\
\hline $\begin{array}{l}\text { Meiner Meinung nach nimmt „Marke“ auf den Social } \\
\text { Media-Plattformen meine Anliegen ernst. }\end{array}$ & 0,925 & 21,919 \\
\hline $\begin{array}{l}\text { Meiner Meinung nach kann sich „Marke“ während der } \\
\text { Kommunikation auf den Social Media-Plattformen gut } \\
\text { in mich hineinversetzen. }\end{array}$ & 0,838 & 19,098 \\
\hline \multicolumn{3}{|c|}{ Kommunikationsinteraktivität (in Anlehnung an McMillan/Hwang 2002) } \\
\hline \multicolumn{3}{|l|}{ Faktorreliabilität $=0,78 ; \mathrm{C} \alpha=0,77 ; \mathrm{DEV}=0,55$} \\
\hline Items & Faktorladung & $t-$ Wert $(>1,64)$ \\
\hline $\begin{array}{l}\text { Beim Auftritt von „Marke“ auf den Social Media-Platt- } \\
\text { formen findet eine Interaktion zwischen der Marke und } \\
\text { mir statt. }\end{array}$ & 0,889 & 16,308 \\
\hline $\begin{array}{l}\text { Beim Auftritt von „Marke“ auf den Social Media-Platt- } \\
\text { formen findet eine wechselseitige Beeinflussung zwi- } \\
\text { schen der Marke und mir statt. }\end{array}$ & 0,744 & 13,903 \\
\hline $\begin{array}{l}\text { Ich empfinde den Auftritt von „Marke“ auf den Social } \\
\text { Media-Plattformen als interaktiv. }\end{array}$ & 0,548 & 10,468 \\
\hline
\end{tabular}




\section{Bruhn/Schäfer/Schoenmüller | Markenkommunikation auf Social Media-Plattformen}

\begin{tabular}{|c|c|c|}
\hline \multicolumn{3}{|c|}{ Kommunikationsmultimedialität (in Anlehnung an Yoo/Donthu 2001) } \\
\hline \multicolumn{3}{|l|}{ Faktorreliabilität $=0,85 ; \mathrm{C} \alpha=0,84 ; \mathrm{DEV}=0,65$} \\
\hline Items & Faktorladung & $\mathrm{t}$-Wert $(>1,64)$ \\
\hline $\begin{array}{l}\text { Die Nutzung von Grafiken wird von „Marke“ auf den } \\
\text { Social Media-Plattformen ansprechend eingesetzt. }\end{array}$ & 0,665 & 13,861 \\
\hline $\begin{array}{l}\text { Das Navigationssystem von „Marke“ auf den Social } \\
\text { Media-Plattformen ist leicht verständlich. }\end{array}$ & 0,807 & 17,372 \\
\hline $\begin{array}{l}\text { Der Auftritt von „Marke“ auf den Social Media-Platt- } \\
\text { formen ist übersichtlich. }\end{array}$ & 0,931 & 20,765 \\
\hline \multicolumn{3}{|c|}{ Markeneinstellung (in Anlehnung an Chattopadhyay/Basu 1990) } \\
\hline \multicolumn{3}{|l|}{ Faktorreliabilität $=0,96 ; \mathrm{C} \alpha=0,96 ; \mathrm{DEV}=0,88$} \\
\hline Items & Faktorladung & t-Wert $(>1,64)$ \\
\hline Insgesamt finde ich „Marke“ gut. & 0,924 & 23,691 \\
\hline Alles in allem mag ich „Marke“. & 0,934 & 24,136 \\
\hline „Marke“ gefällt mir. & 0,961 & 25,384 \\
\hline \multicolumn{3}{|l|}{ Kaufabsicht (in Anlehnung an Dodds/Monroe/Grewal 1991) } \\
\hline \multicolumn{3}{|l|}{ Faktorreliabilität $=0,96 ; \mathrm{C} \alpha=0,96 ; \mathrm{DEV}=0,88$} \\
\hline Items & Faktorladung & $\mathrm{t}$-Wert $(>1,64)$ \\
\hline $\begin{array}{l}\text { Ich kann mir gut vorstellen, in der Zukunft Produkte } \\
\text { von „Marke“ zu kaufen. }\end{array}$ & 0,951 & 24,885 \\
\hline $\begin{array}{l}\text { Falls ich einen Kauf in dieser Produktkategorie tätigen } \\
\text { wollte, würde ich den Erwerb von „Marke“ ernsthaft in } \\
\text { Betracht ziehen. }\end{array}$ & 0,921 & 23,537 \\
\hline $\begin{array}{l}\text { Es ist gut möglich, dass ich in der Zukunft Produkte } \\
\text { von „Marke“ kaufe. }\end{array}$ & 0,943 & 24,541 \\
\hline
\end{tabular}

Tabelle A1: Ergebnisse der Messmodelle 


\begin{tabular}{|l|c|c|c|c|c|c|}
\hline & $\begin{array}{c}\text { Kommuni- } \\
\text { kations- } \\
\text { inhalt }\end{array}$ & $\begin{array}{c}\text { Kommuni- } \\
\text { kations- } \\
\text { beziehung }\end{array}$ & $\begin{array}{c}\text { Kommuni- } \\
\text { kations- } \\
\text { interaktivi- } \\
\text { tät }\end{array}$ & $\begin{array}{c}\text { Kommuni- } \\
\text { kations- } \\
\text { multimedi- } \\
\text { alität }\end{array}$ & $\begin{array}{c}\text { Marken- } \\
\text { einstellung }\end{array}$ & $\begin{array}{c}\text { Kauf- } \\
\text { absicht }\end{array}$ \\
\hline $\begin{array}{l}\text { Kommuni- } \\
\text { kationsin- } \\
\text { halt }\end{array}$ & 0,47 & 0,71 & 0,55 & & & \\
\hline $\begin{array}{l}\text { Kommuni- } \\
\text { kationsbe- } \\
\text { ziehung }\end{array}$ & 0,41 & 0,30 & 0,18 & 0,65 & & \\
\hline $\begin{array}{l}\text { Kommuni- } \\
\text { kationsin- } \\
\text { teraktivität }\end{array}$ & 0,29 & 0,24 & 0,31 & 0,28 & 0,88 & \\
\hline $\begin{array}{l}\text { Kommuni- } \\
\text { kationsmul- } \\
\text { timedialität }\end{array}$ & 0,49 & 0,19 & 0,24 & 0,29 & 0,71 & 0,88 \\
\hline $\begin{array}{l}\text { Marken- } \\
\text { einstellung }\end{array}$ & 0,35 & 0,15 & 0,24 & & \\
\hline $\begin{array}{l}\text { Kauf- } \\
\text { absicht }\end{array}$ & 0,26 & 0 & & & & \\
\hline $\begin{array}{l}\text { In der Diagonalen gefettet stehen die DEV-Werte, unterhalb der Diagonalen die quadrierten } \\
\text { Konstruktkorrelationen. }\end{array}$ &
\end{tabular}

Tabelle A2: Überprüfung der Anforderungskonstrukte auf Diskriminanzvalidität

Manfred Bruhn, Prof. Dr., ist Inhaber des Lehrstuhls für Betriebswirtschaftslehre, insbesondere Marketing und Unternehmensführung, an der Wirtschaftswissenschaftlichen Fakultät der Universität Basel und Honorarprofessor an der Technischen Universität München.

Daniela B. Schäfer, Dipl.-Kffr., und Verena Schoenmüller, Dipl. Kffr., sind wissenschaftliche Mitarbeiterinnen am Lehrstuhl für Betriebswirtschaftslehre, insbesondere Marketing und Unternehmensführung, an der Wirtschaftswissenschaftlichen Fakultät der Universität Basel.

Anschrift: Universität Basel, Wirtschaftswissenschaftliche Fakultät, Lehrstuhl für Marketing und Unternehmensführung, Peter Merian-Weg 6, CH-4002 Basel, Tel.: +41 (0)61/267-32-22, Fax: +41 (0)61/267-28-38, E-Mails: manfred.bruhn@unibas.ch, daniela.schaefer@unibas.ch, verena.schoenmueller@unibas.ch

Die Autoren danken M.Sc. Jan Sedlacek, Goldbach Interactive (Switzerland) AG, für die Unterstützung bei der Datenerhebung. 